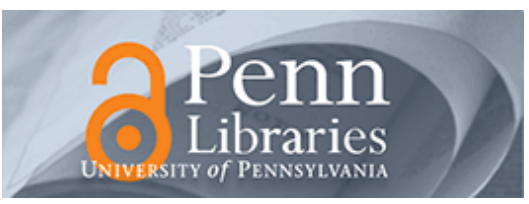

University of Pennsylvania

ScholarlyCommons

$12-2012$

\title{
Game Theoretic Analysis of a Strategic Model of Competitive Contagion and Product Adoption in Social Networks
}

\author{
Arastoo Fazeli \\ University of Pennsylvania \\ Ali Jadbabaie \\ University of Pennsylvania, jadbabai@seas.upenn.edu
}

Follow this and additional works at: https://repository.upenn.edu/ese_papers

Part of the Controls and Control Theory Commons, Dynamic Systems Commons, Other Applied Mathematics Commons, and the Probability Commons

\section{Recommended Citation}

Arastoo Fazeli and Ali Jadbabaie, "Game Theoretic Analysis of a Strategic Model of Competitive Contagion and Product Adoption in Social Networks", . December 2012.

Fazeli, A. \& Jadbabaie, A. (2012). Duopoly Pricing Game in Networks With Local Coordination Effects. Proceedings of the 51st IEEE Conference on Decision and Control, Maui, Hawaii, pp. 74-79.

(c) 2012 IEEE. Personal use of this material is permitted. Permission from IEEE must be obtained for all other uses, in any current or future media, including reprinting/republishing this material for advertising or promotional purposes, creating new collective works, for resale or redistribution to servers or lists, or reuse of any copyrighted component of this work in other works.

This paper is posted at ScholarlyCommons. https://repository.upenn.edu/ese_papers/618

For more information, please contact repository@pobox.upenn.edu. 


\title{
Game Theoretic Analysis of a Strategic Model of Competitive Contagion and Product Adoption in Social Networks
}

\begin{abstract}
In this paper we propose and study a strategic model of marketing and product adoption in social networks. Two firms compete for the spread of their products in a social network. Considering their fixed budgets, they initially determine the payoff of their products and the number of their initial seeds in a network. Afterwards, neighboring agents play a local coordination game over a fixed network which determines the dynamics of the spreading. Assuming myopic best response dynamics, agents choose a product based on the payoff received by actions of their neighbors. This local update dynamics results in a game-theoretic diffusion process in the network. Utilizing earlier results in the literature, we find a lower and an upper bound on the proportion of product adoptions. We derive an explicit characterization of these bounds based on the payoff of products offered by firms, the initial number of adoptions and the underlying structure of the network. We then consider a case in which after switching to the new product, agents might later switch back to the old product with some fixed rate. We show that depending on the rate of switching back to the old product, the new product might always die out in the network eventually. Finally, we consider a game between two firms aiming to optimize their products adoptions while considering their fixed budgets. We describe the Nash equilibrium of this game and show how the optimal payoffs offered by firms and the initial number of seeds depend on the relative budgets of firms.
\end{abstract}

\section{Keywords}

Game Theory, Social Networks, Viral Marketing, Stochastic Process, Optimization

\section{Disciplines}

Controls and Control Theory | Dynamic Systems | Other Applied Mathematics | Probability

\section{Comments}

Fazeli, A. \& Jadbabaie, A. (2012). Duopoly Pricing Game in Networks With Local Coordination Effects. Proceedings of the 51st IEEE Conference on Decision and Control, Maui, Hawaii, pp. 74-79.

(C) 2012 IEEE. Personal use of this material is permitted. Permission from IEEE must be obtained for all other uses, in any current or future media, including reprinting/republishing this material for advertising or promotional purposes, creating new collective works, for resale or redistribution to servers or lists, or reuse of any copyrighted component of this work in other works. 


\title{
Game Theoretic Analysis of a Strategic Model of Competitive Contagion and Product Adoption in Social Networks
}

\author{
Arastoo Fazeli ${ }^{\dagger} \quad$ Ali Jadbabaie ${ }^{\dagger}$
}

\begin{abstract}
In this paper we propose and study a strategic model of marketing and product adoption in social networks. Two firms compete for the spread of their products in a social network. Considering their fixed budgets, they initially determine the payoff of their products and the number of their initial seeds in a network. Afterwards, neighboring agents play a local coordination game over a fixed network which determines the dynamics of the spreading. Assuming myopic best response dynamics, agents choose a product based on the payoff received by actions of their neighbors. This local update dynamics results in a game-theoretic diffusion process in the network. Utilizing earlier results in the literature, we find a lower and an upper bound on the proportion of product adoptions. We derive an explicit characterization of these bounds based on the payoff of products offered by firms, the initial number of adoptions and the underlying structure of the network. We then consider a case in which after switching to the new product, agents might later switch back to the old product with some fixed rate. We show that depending on the rate of switching back to the old product, the new product might always die out in the network eventually. Finally, we consider a game between two firms aiming to optimize their products adoptions while considering their fixed budgets. We describe the Nash equilibrium of this game and show how the optimal payoffs offered by firms and the initial number of seeds depend on the relative budgets of firms.
\end{abstract}

\section{INTRODUCTION}

Recently, there has been a wide range of studies on the role of social networks in shaping individual choices in purchasing new products in networks [1]-[3]. More data from online social networks and advances in information technologies have drawn the attention of firms to exploit this information for their marketing goals. Firms have become interested in models of influence spread in social networks for the promotion of their products and diffusion of their innovations and technologies. The question that arises naturally is how they can use the information about the dynamics of the spread in order to improve their marketing strategies and maximize their profits.

A main feature of product adoption mechanisms is what is often called local coordination effects. This means that when an individual's behavior in adopting a product or a service is a result of her strategic interactions with other people, adopting a common product has a higher payoff. There are diverse sets of examples for such products or services. New technologies and innovations (e.g., cell phones), network goods and services (e.g., fax machines, email accounts), new choices (e.g., choice of languages, TV channels to

\footnotetext{
${ }^{\dagger}$ Department of Electrical and Systems Engineering and GRASP Laboratory at University of Pennsylvania. arastood seas. upenn. edu and jadbabai@seas. upenn. edu. This research was supported by ONR MURI HUNT, ONR MURI N000140810747 and AFOSR Complex Networks Program.
}

watch), online games (e.g., Warcraft), social network web sites (e.g., Facebook, Twitter) and online dating services (e.g., OkCupid) are among many examples in which adoption to a common strategy is more preferable for people. In all these examples, firms might be interested to take the advantage of the social network among people and the local coordination effect of their products and services to trigger a large number of adoptions of their products. Therefore, it might be important for firms to know how to shape their strategies in designing their products and offering them to a set of people in social networks in order to promote their products intelligently and eventually achieving a larger share of the market.

Inspired by the work of Kearns and Goyal in [4], we study strategic competition between two firms which simultaneously allocate their fixed budgets to a set of costumers embedded in a social network. The payoff of firms is the expected number of people adopting their products. This adoption is determined through a game-theoretic diffusion process among costumers in the network. Therefore, a firm shall provide enough incentives for consumption and spread of her product by the payoff that people receive by consuming them. For this purpose and considering their budgets, firms should strategically design their products and know how to initially seed networks.

We propose a game theoretic model of competitive contagion. Two firms initially decide on the amount of investment on their products (which results in the quality of their products and incentive for people to consume them) and the number of agents they initially free offer their products to. Then neighboring agents play a local coordination game which determines the dynamics of the spreading. Assuming a myopic best response dynamics, agents switch between two different products based on the payoff they receive from their neighbors. This local update dynamics results in a gametheoretic diffusion process in the network. Utilizing earlier results in [5] and [6], we find a lower and an upper bound on the number of agents adopting each product at each time. We show that these bounds depend on the payoff of products for agents, initial seeds of the network, and the spectral radius and minimum degree of the adjacency graph. We also consider a case in which agents switch from an old product to a new one based on their myopic best responses, however, they switch back to the old product with some fixed rate. It is shown if the risk dominance of the new product is more than some threshold, the new product will always die out eventually. We then consider a game between firms trying to maximize the lower bound of their products adoptions, while considering their fixed budget. We show that in the Nash equilibrium, the ratio of initial investments of 
firms for producing each unit of products is independent of the network structure and is inversely proportional to their relative budgets. We also show that the ratio of the initial seeds is proportional to the ratio of their budgets.

It is worthwhile to note that the problem of influence and spreading in networks has been extensively studied in the past few years [7]-[13]. Also, diffusion of new behaviors and strategies through local coordination games has been an active field of research [14]-[21]. The main contribution of our work, however, is to explicitly study the tradeoff between investing more money on improving the quality of a product versus seeding it with more people in a social network. The second major contribution of our work is the introduction of the local coordination game dynamics for the problem of competitive contagion in networks. Contrary to the stochastic contagion model in [4], in our model individuals make rational (albeit myopic) choices when adopting a new product. Our results on decay of adoption of one product is also similar to the one obtained in [22] in which the contagion of an action depends on the connectivity distributions of a random network. Our strategic model also bears some similarities to the epidemic model studied in [5], [23].

The rest of this paper is organized as follows: In section II, we introduce our model and dynamics updates for agents playing a local coordination game and find a lower and an upper bound on the spread of products in a network. Then, we consider the case in which agents switch back to the old product with some rate. In section III, we study the game played between firms. Finally, in section IV, we conclude the paper.

\section{THE SPREAD DYNAMICS}

The model considered is based on a game theoretic diffusion model proposed in [24]. There are $n$ agents $V=$ $\{1, \ldots, n\}$ in a social network. The relationship among agents is represented by an undirected graph $G=(V, E)$. Agents $i, j \in V$ are neighbors if $(i, j) \in E$. The adjacency matrix of the graph $G$ is denoted by $A$ where $a_{i j}=1$ if $(i, j) \in E$ and $a_{i j}=0$ otherwise. We assume $a_{i i}=0$ meaning there is no self loop. We denote the minimum degree of the graph $G$ by $d_{\min }$. We assume the graph $G$ is connected, therefore, $d_{\text {min }} \geq 1$. The spectral radius of $G$, is represented by $\rho(G)$, i.e. its largest eigenvalue $\lambda_{1}$ (since graph $G$ is symmetric). We also assume that there are two competing firms $a$ and $b$ producing products $a$ and $b$. These two firms initially offer their products to a set of agents in the network. Let the binary variable $x_{i}(t)$ denotes the choice of agent $i$ at time $t$. We assume $x_{i}(t)=0$ if agent $i$ chooses the product $a$ and $x_{i}(t)=1$ if agent $i$ chooses the product $b$. Therefore, the state of agents at time $t$ is represented by a vector $\vec{x}(t)$. Denote by $s_{0}=\left\{i: x_{i}(0)=0\right\}$ the set of agents who initially are offered the product $a$ and similarly by $s_{1}=\left\{i: x_{i}(0)=1\right\}$ those who initially are offered the product $b$. Denote by $S_{a}$ and $S_{b}$ the cardinality of these sets respectively. These products have some payoffs for neighboring agents depending on their states. If two neighbors in the graph choose the product $a$ they receive a payoff of $p_{a}$, if they both choose the product $b$ they receive a payoff of $p_{b}$ and they receive zero if they choose different products. Therefore, the payoff of the interaction between agent $i$ and agent $j$ can be displayed as the following local coordination game.

\begin{tabular}{|c|c|r|}
\hline & $x_{j}=0$ & $x_{j}=1$ \\
\hline$x_{i}=0$ & $p_{a}$ & 0 \\
\hline$x_{i}=1$ & 0 & $p_{b}$ \\
\hline
\end{tabular}

Thus, the total payoff of an agent is simply the sum of her payoffs obtained from her interactions with her neighbors

$$
u_{i}\left(x_{i}\right)=\sum_{j \in \mathcal{N}_{i}} u_{i}\left(x_{i}, x_{j}\right),
$$

where $\mathcal{N}_{i}$ is the set of neighbors of agent $i$. We assume agents repeatedly apply myopic best response. This means that each agent considering her neighbors, chooses a product that gives her the most payoff. For example, agent $i$ already adopted to the product $a$ switches to the product $b$ if enough of her neighbors have already adopted to the product $b$. For agent $i$ already adopted to the product $a$, the payoff of choosing the product $a$ and $b$ can be written as

$$
\begin{array}{ll}
u_{i}\left(x_{i}=0\right)=p_{a} \sum_{j \in \mathcal{N}_{i}}\left(1-x_{j}\right) & \text { for product } \mathrm{a} \\
u_{i}\left(x_{i}=1\right)=p_{b} \sum_{j \in \mathcal{N}_{i}} x_{j} & \text { for product } \mathrm{b} .
\end{array}
$$

Agent $i$ will switch to the product $b$ if we have $u_{i}\left(x_{i}=0\right)<$ $u_{i}\left(x_{i}=1\right)$ that is

$$
\frac{\sum_{j \in \mathcal{N}_{i}} x_{j}}{d_{i}}>\frac{p_{a}}{p_{a}+p_{b}},
$$

where $d_{i}$ is the number of neighbors of agent $i$ or the cardinality of $\mathcal{N}_{i}$. Similarly, agent $i$ already adopted to the product $b$ will switch to the product $a$ if we have $u_{i}\left(x_{i}=\right.$ 1) $<u_{i}\left(x_{i}=0\right)$ that is

$$
\frac{\sum_{j \in \mathcal{N}_{i}}\left(1-x_{j}\right)}{d_{i}}>\frac{p_{b}}{p_{a}+p_{b}} .
$$

We can define the right hand side of equations (1) and (2) as

$$
r_{a}:=\frac{p_{b}}{p_{a}+p_{b}} \quad r_{b}:=\frac{p_{a}}{p_{a}+p_{b}} .
$$

Note that $r_{a}$ and $r_{b}$ in (3) are the degree of risk dominance of actions $a$ and $b$ respectively. This means that if for agent $i$ already adopted to the product $a$, the fraction of her neighbors adopting to the product $b$ is greater than $r_{b}$, then agent $i$ 's best response is to switch to the product $b$. We can explain $r_{a}$ similarly. This myopic best response dynamics yields to a continuous time stochastic process $\vec{x}(t)$ in which each agent $i$ updates her state upon arrival of a Poisson clock of rate one and switches to the state that gives her the most payoff from interaction with her neighbors. Note that although the rules of updates are deterministic, this process is stochastic due to the randomness in arrival of a random Poisson clock. The result for the bounds of the process $\vec{x}(t)$ with myopic best response dynamics is described in the following lemma. 
Lemma 1: Consider the continuous time process $\vec{x}(t)$ with a random Poisson clock of rate one and the initial condition $\vec{x}(0)$ and dynamics

$$
\begin{aligned}
& x_{i}(t): 0 \rightarrow 1 \text { if } \sum_{j=1}^{n} a_{i j} x_{j}>r_{b} d_{i}, \\
& x_{i}(t): 1 \rightarrow 0 \text { if } \quad \sum_{j=1}^{n} a_{i j}\left(1-x_{j}\right)>r_{a} d_{i} .
\end{aligned}
$$

For this process we have

$$
\begin{aligned}
& 1-\left(\sqrt{\frac{S_{a}}{n}}\right) \exp \left(\left(\frac{\lambda_{1}(G)}{r_{a} d_{\text {min }}}\right) t\right) \leq \frac{\sum_{i=1}^{n} \mathbb{E}\left(x_{i}(t)\right)}{n} \\
& \leq\left(\sqrt{\frac{S_{b}}{n}}\right) \exp \left(\left(\frac{\lambda_{1}(G)}{r_{b} d_{\text {min }}}\right) t\right),
\end{aligned}
$$

where $S_{a}=\left\|\left\{i: x_{i}(0)=0\right\}\right\|$ and $S_{b}=\left\|\left\{i: x_{i}(0)=1\right\}\right\|$ and $r_{a}$ and $r_{b}$ are defined in (3).

Proof: Here we use an approach similar to the one used in [5] and [6]. Consider the continuous time Markov process $\overrightarrow{\tilde{x}}(t)$ with the same initial condition, i.e. $\vec{x}(0)=\overrightarrow{\tilde{x}}(0)$, and

$$
\tilde{x}_{i}(t): 0 \rightarrow 1 \text { at rate } \mathbf{1}\left(\sum_{j=1}^{n} a_{i j} \tilde{x}_{j}(t)>r_{b} d_{i}\right) .
$$

The above expression can be interpreted as

$$
\mathbb{P}\left(\tilde{x}_{i}(t+h)=1 \mid \tilde{x}_{i}(t)=0\right)=\mathbf{1}\left(\sum_{j=1}^{n} a_{i j} \tilde{x}_{j}(t)>r_{b} d_{i}\right) h .
$$

Since $\tilde{x}_{i}(t)$ does not go from one to zero, we can see that $\mathbb{E}\left(x_{i}(t)\right) \leq \mathbb{E}\left(\tilde{x}_{i}(t)\right)$ for all $t \geq 0$. Since the random process $\tilde{x}_{i}(t)$ only takes values of one and zero with some rate, its expected value is the rate of $\tilde{x}_{i}(t)=1$. Therefore, the expected value of the random process $\tilde{x}_{i}(t)$ is

$$
\mathbb{E}\left(\tilde{x}_{i}(t+h)\right)=\mathbf{1}\left(\sum_{j=1}^{n} a_{i j} \tilde{x}_{j}(t)>r_{b} d_{i}\right) h .
$$

Now define the Markov process $\vec{y}(t)$ with $\vec{x}(0)=\vec{y}(0)$

$$
y_{i}(t): k \rightarrow k+1 \quad \text { at rate } \frac{\sum_{j=1}^{n} a_{i j} y_{j}(t)}{r_{b} d_{\text {min }}} .
$$

This means that

$$
\mathbb{P}\left(y_{i}(t+h)=k+1 \mid y_{i}(t)=k\right)=\frac{\sum_{j=1}^{n} a_{i j} y_{j}(t)}{r_{b} d_{\text {min }}} h,
$$

and as a result

$$
\mathbb{E}\left(y_{i}(t+h)\right)-\mathbb{E}\left(y_{i}(t)\right)=\frac{\sum_{j=1}^{n} a_{i j} y_{j}(t)}{r_{b} d_{\min }} h .
$$

Since $\mathbf{1}\left(\sum_{j=1}^{n} a_{i j} y_{j}(t)>r_{b} d_{i}\right)<\frac{\sum_{j=1}^{n} a_{i j} y_{j}(t)}{r_{b} d_{m i n}}$, standard coupling arguments implies $\mathbb{E}\left(\tilde{x}_{i}(t)\right) \leq \mathbb{E}\left(y_{i}(t)\right)$. Now since $\mathbb{E}\left(x_{i}(t)\right) \leq \mathbb{E}\left(\tilde{x}_{i}(t)\right)$ we can get $\mathbb{E}\left(x_{i}(t)\right) \leq \mathbb{E}\left(y_{i}(t)\right)$ and as a result $\sum_{i=1}^{n} \mathbb{E}\left(x_{i}(t)\right) \leq \sum_{i=1}^{n} \mathbb{E}\left(y_{i}(t)\right)$. Notice that the process $\mathbb{E}\left(y_{i}(t)\right)$ takes value in $\mathbb{R}$. Therefore, we finally get the following differential equation for $\mathbb{E}\left(y_{i}(t)\right)$

$$
\frac{d}{d t} \mathbb{E}(\vec{y}(t))=\left(\frac{A}{r_{b} d_{\text {min }}}\right) \mathbb{E}(\vec{y}(t))
$$

Computing the solution, we have

$$
\mathbb{E}(\vec{y}(t))=\exp \left(\left(\frac{A}{r_{b} d_{\text {min }}}\right) \mathbb{E}(\vec{y}(0)) .\right.
$$

Since $A$ is symmetric, $\exp \left(\left(\frac{A}{r_{b} d_{\text {min }}}\right) t\right)$ is also symmetric and its largest eigenvalue is equal to $\exp \left(\left(\frac{\lambda_{1}(G)}{r_{b} d_{\text {min }}}\right) t\right)$. Therefore,

$$
\|\mathbb{E}(\vec{y}(t))\|_{2} \leq \exp \left(\left(\frac{\lambda_{1}(G)}{r_{b} d_{\min }}\right) t\right)\|\mathbb{E}(\vec{y}(0))\|_{2} .
$$

Employing Cauchy-Schwarz inequality we get

$$
\sum_{i=1}^{n} \mathbb{E}\left(y_{i}(t)\right) \leq\|\mathbb{E}(\vec{y}(t))\|_{2}\left\|\mathbf{1}_{n}\right\|_{2}=\|\mathbb{E}(\vec{y}(t))\|_{2} \sqrt{n} .
$$

Simplifying further, we obtain

$$
\begin{aligned}
& \sum_{i=1}^{n} \mathbb{E}\left(x_{i}(t)\right) \leq \sum_{i=1}^{n} \mathbb{E}\left(y_{i}(t)\right) \\
& \leq \sqrt{n} \exp \left(\left(\frac{\lambda_{1}(G)}{r_{b} d_{\text {min }}}\right) t\right)\|(\vec{y}(0))\|_{2} .
\end{aligned}
$$

Note that $\vec{x}(0)=\vec{y}(0)$ and $x_{i}^{2}(0)=x_{i}(0)$ since $x_{i} \in\{0,1\}$. Therefore, we get

$$
\sum_{i=1}^{n} \mathbb{E}\left(x_{i}(t)\right) \leq \sqrt{n} \exp \left(\left(\frac{\lambda_{1}(G)}{r_{b} d_{\min }}\right) t\right) \sqrt{\sum_{i=1}^{n} x_{i}(0)} .
$$

Hence, the expected number of adoptions to the product $a$ is bounded by

$$
\frac{\sum_{i=1}^{n} \mathbb{E}\left(x_{i}(t)\right)}{n} \leq\left(\sqrt{\frac{S_{b}}{n}}\right) \exp \left(\left(\frac{\lambda_{1}(G)}{r_{b} d_{\text {min }}}\right) t\right),
$$

where we used the definition of $S_{b}=\left\|\left\{i: x_{i}(0)=1\right\}\right\|$. Now consider the continuous time stochastic process $\vec{z}(t)$ with $\vec{z}(0)=\vec{x}(0)$ and transition rate

$$
z_{i}(t): k \rightarrow k-1 \quad \text { at rate } \frac{\sum_{j=1}^{n} a_{i j}\left(1-z_{j}(t)\right)}{r_{a} d_{\text {min }}} .
$$

Since this process is only decreasing and $\mathbf{1}\left(\sum_{j=1}^{n} a_{i j}(1-\right.$ $\left.\left.z_{j}(t)\right)>r_{a} d_{i}\right)<\frac{\sum_{j=1}^{n} a_{i j}\left(1-z_{j}(t)\right)}{r_{a} d_{\min }}$, similar to what we did for the process $\vec{y}(t)$ and using coupling arguments, it follows that $\sum_{i=1}^{n} \mathbb{E}\left(z_{i}(t)\right) \leq \sum_{i=1}^{n} \mathbb{E}\left(x_{i}(t)\right)$. From the transition rates for process $\vec{z}(t)$ we get

$$
\frac{d}{d t} \mathbb{E}(\vec{z}(t))=-\left(\frac{A}{r_{a} d_{\text {min }}}\right) \mathbb{E}(\overrightarrow{1}-\vec{z}(t)) .
$$

Therefore,

$$
\frac{d}{d t} \mathbb{E}(\overrightarrow{1}-\vec{z}(t))=\left(\frac{A}{r_{a} d_{\text {min }}}\right) \mathbb{E}(\overrightarrow{1}-\vec{z}(t)) .
$$

Define process $\vec{w}(t)=\overrightarrow{1}-\vec{z}(t)$. The dynamics can now be written as

$$
\frac{d}{d t} \mathbb{E}(\vec{w}(t))=\left(\frac{A}{r_{a} d_{\min }}\right) \mathbb{E}(\vec{w}(t)) .
$$

Using the same arguments that we did for the process $\vec{y}(t)$, we get the following solution

$$
\mathbb{E}(\vec{w}(t))=\exp \left(\left(\frac{A}{r_{a} d_{\text {min }}}\right) \mathbb{E}(\vec{w}(0)) .\right.
$$


The above equation results in the following bound

$$
\frac{\sum_{i=1}^{n} \mathbb{E}\left(w_{i}(t)\right)}{n} \leq\left(\sqrt{\frac{\sum_{i=1}^{n} w_{i}(0)}{n}}\right) \exp \left(\left(\frac{\lambda_{1}(G)}{r_{a} d_{\min }}\right) t\right) .
$$

Note that $\vec{w}(0)=\overrightarrow{1}-\vec{z}(0)=\overrightarrow{1}-\vec{x}(0)$. Therefore, using the definition of $S_{a}=\left\|\left\{i: x_{i}(0)=0\right\}\right\|$ we get

$$
\frac{\sum_{i=1}^{n} \mathbb{E}\left(w_{i}(t)\right)}{n} \leq\left(\sqrt{\frac{S_{a}}{n}}\right) \exp \left(\left(\frac{\lambda_{1}(G)}{r_{a} d_{\min }}\right) t\right)
$$

Now since $\vec{w}(t)=\overrightarrow{1}-\vec{z}(t)$ and $\mathbb{E}(\vec{z}(t)) \leq \mathbb{E}(\vec{x}(t))$, using the above equation we obtain

$$
1-\left(\sqrt{\frac{S_{a}}{n}}\right) \exp \left(\left(\frac{\lambda_{1}(G)}{r_{a} d_{\text {min }}}\right) t\right) \leq \frac{\sum_{i=1}^{n} \mathbb{E}\left(x_{i}(t)\right)}{n} .
$$

Equation (4) and (5) implies

$$
\begin{aligned}
& 1-\left(\sqrt{\frac{S_{a}}{n}}\right) \exp \left(\left(\frac{\lambda_{1}(G)}{r_{a} d_{\text {min }}}\right) t\right) \leq \frac{\sum_{i=1}^{n} \mathbb{E}\left(x_{i}(t)\right)}{n} \\
& \leq\left(\sqrt{\frac{S_{b}}{n}}\right) \exp \left(\left(\frac{\lambda_{1}(G)}{r_{b} d_{\text {min }}}\right) t\right) .
\end{aligned}
$$

The above result states that the expected value of the fraction of agents adopting the product $b$ increases (decreases) at most (at least) exponentially in time. Note that as time goes to infinity, these bounds become loose, however, we study the problem in a fixed time horizon $T$ where we can still exploit these bounds. Also, notice that when $S_{b}=0$, the upper bound in (6) becomes zero meaning that the fraction always remains zero. This makes sense because when $S_{b}=0$, there is no agent with action $x_{i}=1$ to spread it in the network. Similarly, when $S_{a}=0$, the lower bound in (6) becomes one meaning that the fraction always remains one. This is also due to the fact that when $S_{a}=0$, there is no agent with action $x_{i}=0$ to spread it in the network. Equation (6) also implies

$$
\begin{aligned}
& 1-\left(\sqrt{\frac{S_{b}}{n}}\right) \exp \left(\left(\frac{\lambda_{1}(G)}{r_{b} d_{\min }}\right) t\right) \leq \frac{\sum_{i=1}^{n}\left(1-\mathbb{E}\left(x_{i}(t)\right)\right)}{n} \\
& \leq\left(\sqrt{\frac{S_{a}}{n}}\right) \exp \left(\left(\frac{\lambda_{1}(G)}{r_{a} d_{\min }}\right) t\right) .
\end{aligned}
$$

Therefore, the expected value of the fraction of agents adopting the product $a$ also increases (decreases) at most (at least) exponentially in time. Now we consider a case in which employing myopic best responses, agents switch from the product $a$ to the product $b$, however, they switch back to the old product with some fixed rate $\beta$. This could be due to the realized inferiority of the new product $b$ compared to the old product $a$. In the following lemma we study a condition under which the new product will eventually die out.

Lemma 2: Consider the continuous stochastic process $\vec{x}(t)$ in which each agent with state $x_{i}(t)=0$ updates her state upon arrival of a Poisson clock of rate one and switches to state $x_{i}(t)=1$ if $u_{i}\left(x_{i}=0\right)<u_{i}\left(x_{i}=1\right)$ and holds on to state $x_{i}(t)=0$ otherwise. However, each agent with state $x_{i}(t)=1$ switches to state $x_{i}=0$ with some fixed rate $\beta$.
If $\frac{\lambda_{1}(G)}{r_{b} d_{\min }}<\beta$, then for any initial condition $x(0)$ we will have

$$
\lim _{t \rightarrow \infty} \mathbb{P}\left(\sum_{i=1}^{n} x_{i}(t)>0\right)=0
$$

Proof: Define the continuous time stochastic process $\vec{v}(t)$ with $\vec{v}(0)=\vec{x}(0)$ and transition rate

$$
\begin{array}{ll}
v_{i}(t): 0 \rightarrow 1 \quad \text { at rate } & \frac{\sum_{j=1}^{n} a_{i j} v_{j}(t)}{r_{b} d_{\min }}, \\
v_{i}(t): 1 \rightarrow 0 & \text { at rate }
\end{array}
$$

For this process using similar approach we did earlier we can get

$$
\frac{d}{d t} \mathbb{E}(\vec{v}(t))=\left(\frac{A}{r_{b} d_{\text {min }}}-\beta I\right) \mathbb{E}(\vec{v}(t)),
$$

where $I$ is the identity matrix. Therefore, similar to before we will have

$$
\begin{aligned}
& \sum_{i=1}^{n} \mathbb{E}\left(x_{i}(t)\right) \leq \sum_{i=1}^{n} \mathbb{E}\left(v_{i}(t)\right) \\
& \leq \sqrt{n} \exp \left(\left(\frac{\lambda_{1}(G)}{r_{b} d_{\text {min }}}-\beta\right) t\right)\|(\vec{v}(0))\|_{2} .
\end{aligned}
$$

Since $\|(\vec{v}(0))\|_{2} \leq \sqrt{n}$, we have

$$
\frac{\sum_{i=1}^{n} \mathbb{E}\left(v_{i}(t)\right)}{n} \leq \exp \left(\left(\frac{\lambda_{1}(G)}{r_{b} d_{\text {min }}}-\beta\right) t\right) .
$$

Note that $\mathbb{E}(\vec{x}(t)) \leq \mathbb{E}(\vec{v}(t))$ implies that

$$
\mathbb{P}\left(\sum_{i=1}^{n} x_{i}(t)=0\right) \geq \mathbb{P}\left(\sum_{i=1}^{n} v_{i}(t)=0\right) .
$$

Therefore,

$$
\begin{aligned}
& \mathbb{P}\left(\sum_{i=1}^{n} x_{i}(t)>0\right)=1-\mathbb{P}\left(\sum_{i=1}^{n} x_{i}(t)=0\right) \\
& <1-\mathbb{P}\left(\sum_{i=1}^{n} v_{i}(t)=0\right)=\mathbb{P}\left(\sum_{i=1}^{n} v_{i}(t)>0\right) .
\end{aligned}
$$

Moreover, we have

$$
\mathbb{P}\left(\sum_{i=1}^{n} v_{i}(t)>0\right) \leq \sum_{i=1}^{n} \mathbb{E}\left(v_{i}(t)\right)
$$

The above inequality together with (7) and (8) implies

$$
\mathbb{P}\left(\sum_{i=1}^{n} x_{i}(t)>0\right) \leq n \exp \left(\left(\frac{\lambda_{1}(G)}{r_{b} d_{\min }}-\beta\right) t\right) .
$$

Therefore, if

$$
\frac{\lambda_{1}(G)}{r_{b} d_{\min }}<\beta
$$

we have

$$
\lim _{t \rightarrow \infty} \mathbb{P}\left(\sum_{i=1}^{n} x_{i}(t)>0\right)=0
$$

The above result implies that if $\frac{\lambda_{1}(G)}{r_{b} d_{\min }}<\beta$ the new product $b$ dies out eventually and cannot propagate in the network. Note that if we interpret $\beta$ as the rate of an infected node being cured, this condition would become similar to the 
condition for an epidemic to die out eventually. Using the definition of $r_{b}$ in (3), the condition in equation (9) for the new product to die out can also be written as

$$
r_{b}>\frac{\lambda_{1}(G)}{\beta d_{\text {min }}} \text {. }
$$

The above result implies that if the degree of risk dominance of the new product $b$ is greater than some threshold, then the product dies out eventually in the network. In other words, if the fraction of an agent's neighbors required to choose product $b$ in order to make product $b$ preferred over the product $a$ is above some threshold, then the product $b$ cannot spread in the network. Also, from equation (9) we can get

$$
\frac{p_{b}}{p_{a}}<\frac{\beta d_{\min }}{\lambda_{1}(G)}-1
$$

The interpretation of the above condition is that if the relative payoff of the new product $b$ compared to the old product $a$ is less than some threshold then the new product dies out eventually. This condition also implies that a necessary condition for the new product to die out is that the rate of switching back to the old product is greater than $\frac{\lambda_{1}(G)}{d_{\text {min }}}$.

In the next section we discuss how firms can exploit this information in order to maximize the spread of their products and also find the Nash equilibrium of the game played between two firms.

\section{NASh EQUiLIBRIUM ANALYSiS}

In this section we study how firms can maximize the spread of their products and also describe the Nash equilibrium of the game played between two firms. As we mentioned earlier, the goal of these two competing firms is to maximize the spread of their products in the network. However, they will have to consider their budget as well. As we mentioned in section II, initially they offer their products to a subset of agents. This can be viewed as an initial free offer to seed the network and promote their products. We assume offering each unit of product $a$ and $b$ costs $c_{a}$ and $c_{b}$ for firms $a$ and $b$ respectively. We also assume that the payoff of products for agents in the social network is a function of the costs of firms. This is a standard assumption, as in order to produce higher quality products for agents in a social network, firms would have to spend more money on their products. Therefore, we assume payoffs are implicit functions of costs or in other words $p_{a}=p_{a}\left(c_{a}\right)$ and $p_{b}=p_{b}\left(c_{b}\right)$. Considering a fixed finite time horizon, each firm tries to maximize the lower bound of her product adoptions. Hence, firms solve the following optimization problems

$$
\begin{aligned}
& \max _{c_{a} \geq 0} U_{a}\left(p_{a}\left(c_{a}\right), p_{b}\left(c_{b}\right)\right)=1-\left(\sqrt{\frac{S_{b}}{n}}\right) \exp \left(\left(\frac{\lambda_{1}(G)}{r_{b} d_{\min }}\right) t\right) \\
& \text { Subject to } S_{a} c_{a}=K_{a}, \\
& \max _{c_{b} \geq 0} U_{b}\left(p_{a}\left(c_{a}\right), p_{b}\left(c_{b}\right)\right)=1-\left(\sqrt{\frac{S_{a}}{n}}\right) \exp \left(\left(\frac{\lambda_{1}(G)}{r_{a} d_{\text {min }}}\right) t\right)
\end{aligned}
$$$$
\text { Subject to } S_{b} c_{b}=K_{b} \text {, }
$$

where $K_{a}$ and $K_{b}$ are the total budgets of firms $a$ and $b$ to initially seed the network. Here for firm $a$ (or $b$ ), one of variables $c_{a}$ or $S_{a}$ is the independent variable and the other one is determined by that in a way that $S_{a} c_{a}=K_{a}$. In the following theorem we describe the Nash equilibrium of the game played between firms.

Theorem 1: Consider firms $a$ and $b$ with utility functions $U_{a}$ and $U_{b}$ as described in (11). If for payoff of products we assume Cobb-Douglas function, i.e. $p(c)=d l^{\beta} c^{\alpha}$, then in the Nash equilibrium of the game between firms we have

$$
\frac{c_{b}^{2 \alpha_{b}-1}}{c_{a}^{2 \alpha_{a}-1}}=\left(\frac{K_{a}}{K_{b}}\right)\left(\frac{\alpha_{b}}{\alpha_{a}}\right)\left(\frac{d_{a}}{d_{b}}\right)^{2}\left(\frac{l_{a}^{\beta_{a}}}{l_{b}^{\beta_{b}}}\right)^{2},
$$

also when firms have the same constants in their payoff functions we obtain

$$
\frac{c_{b}^{*}}{c_{a}^{*}}=\left(\frac{K_{a}}{K_{b}}\right)^{\frac{1}{2 \alpha-1}}, \quad \frac{S_{b}^{*}}{S_{a}^{*}}=\left(\frac{K_{b}}{K_{a}}\right)^{\frac{2 \alpha}{2 \alpha-1}} .
$$

Proof: ${ }^{a}$ First note that in Cobb-Douglas production function, $c$ is the capital input (cost of the firm for producing each unit of the product) $l$ is the labor input, $d$ is total factor productivity and $\alpha$ and $\beta$ are the output elasticities of labor and capital respectively which are determined by available technology. Here, we assume the payoff is a function of the cost only, i.e. $p=p(c)$, meaning that other parameters are fixed. Now notice that optimization problems in (11) can be written as

$$
\min _{c_{a} \geq 0} U_{a}\left(p_{a}\left(c_{a}\right), p_{b}\left(c_{b}\right)\right)=\left(\sqrt{\frac{S_{b}}{n}}\right) \exp \left(\left(\frac{\lambda_{1}(G)}{r_{b} d_{\text {min }}}\right) t\right)
$$

Subject to $S_{a} c_{a}=K_{a}$

$$
\min _{c_{b} \geq 0} U_{b}\left(p_{a}\left(c_{a}\right), p_{b}\left(c_{b}\right)\right)=\left(\sqrt{\frac{S_{a}}{n}}\right) \exp \left(\left(\frac{\lambda_{1}(G)}{r_{a} d_{\text {min }}}\right) t\right)
$$

Subject to $S_{b} c_{b}=K_{b}$.

Instead of minimizing $U_{a}$ and $U_{b}$, since the logarithm function is monotone, we can minimize $\log \left(U_{a}\right)$ and $\log \left(U_{b}\right)$. Using the fact that $S_{a}=\frac{K_{a}}{c_{a}}, S_{b}=\frac{K_{b}}{c_{b}}, n=S_{a}+S_{b}$ and using the definition of $r_{a}$ and $r_{b}$ in (3), after simplification we get

$$
\begin{aligned}
& \log \left(U_{a}\right)=\left(\frac{1}{2}\right) \log \left(\frac{\frac{K_{b}}{c_{b}}}{\frac{K_{a}}{c_{a}}+\frac{K_{b}}{c_{b}}}\right)+\left(\frac{\lambda_{1}(G) t}{d_{\text {min }}}\right)\left(\frac{p_{a}+p_{b}}{p_{a}}\right) \\
& \log \left(U_{b}\right)=\left(\frac{1}{2}\right) \log \left(\frac{\frac{K_{a}}{c_{a}}}{\frac{K_{a}}{c_{a}}+\frac{K_{b}}{c_{b}}}\right)+\left(\frac{\lambda_{1}(G) t}{d_{\text {min }}}\right)\left(\frac{p_{a}+p_{b}}{p_{b}}\right) .
\end{aligned}
$$

In order to find the best response of firms $a$ and $b$, i.e. the optimal cost of a firm conditioned on the cost of the other firm, we should take the derivative of $\log \left(U_{a}\right)$ and $\log \left(U_{a}\right)$ with respect to $c_{a}$ and $c_{b}$ and set them to zero. After doing this step and simplification we obtain

$$
\begin{gathered}
\frac{K_{a} c_{b}}{2 c_{a}\left(K_{a} c_{b}+K_{b} c_{a}\right)}=\frac{\alpha_{a} d_{b} l_{b}^{\beta_{b}} c_{b}^{\alpha_{b}} t}{d_{a} l_{a}^{\beta_{a}} c_{a}^{\alpha_{a}+1}}, \\
\frac{K_{b} c_{a}}{2 c_{b}\left(K_{a} c_{b}+K_{b} c_{a}\right)}=\frac{\alpha_{b} d_{a} l_{a}^{\beta_{a}} c_{a}^{\alpha_{a}} t}{d_{b} l_{b}^{\beta_{b}} c_{b}^{\alpha_{b}+1}} .
\end{gathered}
$$

Dividing two equations in (12) by each other and after further simplification we have

$$
\frac{c_{b}^{2 \alpha_{b}-1}}{c_{a}^{2 \alpha_{a}-1}}=\left(\frac{K_{a}}{K_{b}}\right)\left(\frac{\alpha_{b}}{\alpha_{a}}\right)\left(\frac{d_{a}}{d_{b}}\right)^{2}\left(\frac{l_{a}^{\beta_{a}}}{l_{b}^{\beta_{b}}}\right)^{2} .
$$


Here we can see that in the Nash equilibrium, firms with more budget, labor input and total factor productivity invest less capital on their product. However, firms with more output elasticity of capital spend more money on their production. If we assume firms have the same labor and the same constants in their payoff functions we obtain

$$
\frac{c_{b}^{*}}{c_{a}^{*}}=\left(\frac{K_{a}}{K_{b}}\right)^{\frac{1}{2 \alpha-1}},
$$

and for initial seeds we get

$$
\frac{S_{b}^{*}}{S_{a}^{*}}=\left(\frac{K_{b}}{K_{a}}\right)^{\frac{2 \alpha}{2 \alpha-1}} .
$$

If we also assume $\alpha=1$, i.e. payoff is a linear function of the cost, we will have

$$
\frac{c_{b}^{*}}{c_{a}^{*}}=\frac{K_{a}}{K_{b}}, \quad \frac{S_{b}^{*}}{S_{a}^{*}}=\left(\frac{K_{b}}{K_{a}}\right)^{2} .
$$

As we can see the strategy of firms for spending their budget is independent of the time horizon that their optimization is defined. Also it is worth mentioning that relative initial seeds and initial investments of firms only depend on the relative budget of firms and is independent of the network structure. This result describes the tradeoff between investment on improving the quality of a product versus the initial seed of a product in a social network. The above analysis provides a crucial insight for firms marketing strategy on how firms should allocate their fixed budget in order to maximize the adoption of their products. The interpretation of this result for new marketers is that if they have a bigger budget than an incumbent firm, they should seed a larger number of people while lowering their investment on their product and as a result the quality of their product. On the other hand, if they have a small budget compared to an incumbent firm, they will be better off focusing on the quality of their product rather than seeding it on a large portion of the network. The relative importance of the initial investment compared to the initial seed depends on parameters of a firm's production function.

\section{CONCLUSION}

In this paper we proposed and studied a strategic model of product adoption in a social network. In this model, two firms compete for maximizing the adoptions of their products in a social network. Considering their fixed budgets, they initially decide on the capital they invest on their products and also the number of people they free offer their products to. The dynamics of the spread is determined by a local coordination game among agents in which agents act myopic rationally to maximize their profits. We found a lower and an upper bound on the proportion of products adoptions based on the payoff of products offered by firms, the initial number of adoptions and the underlying structure of the network. We also proposed conditions on how a new product might die out in a network eventually. Finally, we characterized the Nash equilibrium of the game between firms. We showed that in the Nash equilibrium the ratio of capital inputs of firms and initial seeds is independent of the network structure and is only a function of their relative budgets. We also showed that firms with bigger budget than an incumbent firm should seed a large number of people while lowering their investment on their products. On the other hand, when they have a small budget compared to an incumbent firm, they are better off focusing on the quality of their product rather than seeding it on a large portion of the network. As a future direction, we plan to look at the case where the choice of seeding can be different based on the location of each agent and analyze the equilibrium in that setting.

\section{REFERENCES}

[1] L. Feick and L. Price, "The market maven: A diffuser of marketplace information," The Journal of Marketing, pp. 83-97, 1987.

[2] P. Reingen, B. Foster, J. Brown, and S. Seidman, "Brand congruence in interpersonal relations: A social network analysis," Journal of Consumer Research, pp. 771-783, 1984.

[3] D. Godes and D. Mayzlin, "Using online conversations to study wordof-mouth communication," Marketing Science, pp. 545-560, 2004.

[4] S. Goyal and M. Kearns, "Competitive contagion in networks," 2011.

[5] A. Ganesh, L. Massoulié, and D. Towsley, "The effect of network topology on the spread of epidemics," in INFOCOM 2005. 24th Annual Joint Conference of the IEEE Computer and Communications Societies. Proceedings IEEE, vol. 2, pp. 1455-1466, IEEE, 2005.

[6] H. Amini, M. Draief, and M. Lelarge, "Marketing in a random network," Network Control and Optimization, pp. 17-25, 2009.

[7] C. Ballester, A. Calvó-Armengol, and Y. Zenou, "Who's who in networks. wanted: the key player," Econometrica, vol. 74, no. 5, pp. 1403-1417, 2006.

[8] S. Bharathi, D. Kempe, and M. Salek, "Competitive influence maximization in social networks," Internet and Network Economics, pp. 306-311, 2007.

[9] A. Galeotti and S. Goyal, "Influencing the influencers: a theory of strategic diffusion," The RAND Journal of Economics, vol. 40, no. 3, pp. 509-532, 2009.

[10] D. Kempe, J. Kleinberg, and É. Tardos, "Maximizing the spread of influence through a social network," in Proceedings of the ninth ACM SIGKDD international conference on Knowledge discovery and data mining, pp. 137-146, ACM, 2003.

[11] D. Kempe, J. Kleinberg, and É. Tardos, "Influential nodes in a diffusion model for social networks," Automata, Languages and Programming, pp. 99-99, 2005.

[12] G. Chasparis and J. Shamma, "Control of preferences in social networks," in Decision and Control (CDC), 2010 49th IEEE Conference on, pp. 6651-6656, IEEE, 2010.

[13] A. Vetta, "Nash equilibria in competitive societies, with applications to facility location, traffic routing and auctions," in Foundations of Computer Science, 2002. Proceedings. The 43rd Annual IEEE Symposium on, pp. 416-425, IEEE, 2002.

[14] G. Ellison, "Learning, local interaction, and coordination," Econometrica: Journal of the Econometric Society, pp. 1047-1071, 1993.

[15] M. Kandori, G. Mailath, and R. Rob, "Learning, mutation, and long run equilibria in games," Econometrica: Journal of the Econometric Society, pp. 29-56, 1993.

[16] J. Harsanyi and R. Selten, "A general theory of equilibrium selection in games," MIT Press Books, vol. 1, 1988.

[17] H. Young, "The evolution of conventions," Econometrica: Journal of the Econometric Society, pp. 57-84, 1993.

[18] H. Young, Individual strategy and social structure: An evolutionary theory of institutions. Princeton Univ Pr, 2001.

[19] H. Young, "The diffusion of innovations in social networks," The Economy As an Evolving Complex System III: Current Perspectives and Future Directions, p. 267, 2006.

[20] A. Montanari and A. Saberi, "The spread of innovations in social networks," Proceedings of the National Academy of Sciences, vol. 107, no. 47 , p. $20196,2010$.

[21] J. Kleinberg, "Cascading behavior in networks: Algorithmic and economic issues," Algorithmic game theory, pp. 613-632, 2007.

[22] D. López-Pintado, "Contagion and coordination in random networks," International Journal of Game Theory, vol. 34, no. 3, pp. 371-381, 2006.

[23] M. Draief, A. Ganesh, and L. Massoulié, "Thresholds for virus spread on networks," in Proceedings of the 1st international conference on Performance evaluation methodolgies and tools, p. 51, ACM, 2006.

[24] S. Morris, "Contagion," The Review of Economic Studies, vol. 67, no. 1, p. 57, 2000. 